\title{
Los diálogos reconstruidos en la entrevista sociolingüística
}

\author{
María José Gallucci ${ }^{1}$ \\ Universidad de Navarra
}

\begin{abstract}
Resumen
En este artículo describimos y explicamos de qué manera se construyen los diálogos reconstruidos (DREC) en un conjunto de muestras orales del PRESEEA-Caracas (Bentivoglio y Malaver 2006, 2012; Gallucci, González y Malaver 2013). En esta primera aproximación al estudio de esta instancia polifónica en el corpus del español caraqueño, hemos considerado los tipos de citas que conforman los DREC, el marco introductor, los turnos, los enunciadores o speakers y el uso de los DREC en relación con los factores sociales inherentes a nuestra muestra de estudio.

Los resultados generales obtenidos muestran que: i) los DREC están conformados, sobre todo, por citas directas sin marco o freestanding quotations (Cameron 1998); ii) suelen ir introducidos por citas directas tradicionales, es decir, encabezadas por un verbo de comunicación conjugado; iii) en su mayoría están constituidos por secuencias organizadas en pares de pregunta/respuesta o de aserción/ réplica; iv) el locutor suele animar, en su mayoría, dos voces, incluida
\end{abstract}


la propia. En lo que respecta a las variables extralingüísticas, los datos estadísticos muestran que el uso de enunciados citativos en el marco de un DREC guarda relación, al menos en nuestras entrevistas, con el sexo y el grado de instrucción de los hablantes.

Palabras clave: discurso referido, diálogos reconstruidos, metapragmática, análisis del discurso, sociolingüística.

\title{
RECONSTRUCTED DIALOGUES IN THE SOCIOLINGUISTIC INTERVIEW
}

\begin{abstract}
In this article, we report the analysis of reconstructed dialogues (DREC) in a sample of the socially-stratified corpus PRESEEACaracas (Bentivoglio \& Malaver 2006, 2012; Gallucci, González \& Malaver 2013). In this first approach to the use of DREC as a polyphonic instance in Spanish spoken in Caracas, we identified several features in these dialogues: types of quotes, introductory framework, turn-taking configuration, and type of narrator. Moreover, we also examined the potential relationship between DRECs and social variables (i.e., gender, age and level of instruction). Results show i) that DRECs are usually constituted by cases of direct speech without a verb or freestanding quotations (Cameron 1998); ii) that the introductory frame of DRECs tends to be an instance of direct speech headed by a communication verb, iii) that the sequences of the dialogues are organized in pairs of question/answer or assertion /response; iv) that the narrator animates two voices, including their own. Regarding extralinguistic variables, we found that the use of DRECs in our sample was associated with the gender of the speakers and their level of instruction.
\end{abstract}

Key words: reported speech, constructed dialogues, metapragmatics, discourse analysis, sociolinguistics.

Recibido: $21 / 04 / 20$

Aceptado: 18/01/21 


\section{INTRODUCCIÓN ${ }^{2}$}

En los últimos años, han aumentado de manera considerable los estudios empíricos sobre el discurso referido (DR) en la oralidad (Fernández 2012; Gallucci 2013, 2014, 2018, 2019; San Martín y Guerrero 2013; Benavent Payá 2015; Gallucci y Vargas 2015; San Martín 2015; Estévez 2016; Grajales 2017; Klee y Caravedo 2017, Repede 2018). Las investigaciones recientes incluyen, por lo general, consideraciones pragmáticas y sociolingüísticas a propósito del objeto de estudio. Sin embargo, llama la atención que, como señala Camargo (2007-2008: 50), a pesar de que los estudios sobre el diálogo continúan siendo un centro de interés para los analistas del discurso y de la conversación, desde esta perspectiva aún son escasos los estudios sobre la estructura y el funcionamiento de los diálogos reconstruidos (DREC), instancia polifónica muy habitual en el interior de la narración oral conversacional (Tannen 1986, 1989) cuyos turnos se construyen, precisamente, a partir de enunciados de $\mathrm{DR}^{3}$.

En consonancia con lo anterior, esta investigación piloto tiene como objetivo general analizar cómo se caracterizan los DREC en un conjunto de muestras orales del PRESEEA-Caracas estratificadas según la edad, el sexo - o género-y el grado de instrucción de los hablantes.

Las preguntas que nos hemos planteado son las siguientes: i) ¿cuáles son los tipos de citas que se emplean en estos diálogos?, ii) ¿cómo se introducen?, iii) ¿de qué manera se construyen los turnos de habla?, iv) ¿cuántas voces se animan? y v) ¿estos diálogos guardan alguna relación con los factores sociales antes descritos?

2 Este artículo forma parte de un estudio de mayor alcance sobre el discurso referido en muestras orales del español americano ( $c f$. Gallucci 2018). Asimismo, se inscribe en el Grupo de Investigación sobre Oralidad (UNoral) de la Universidad de Navarra.

Agradecemos a los evaluadores del artículo sus pertinentes observaciones sobre el texto.

Sin embargo, desde un enfoque cognitivo destacan los trabajos de Pascual (2014) y de Pascual y Sandler (2016) a propósito de las interacciones fictivas. 


\section{EL DISCURSO REFERIDO COMO CONTINUUM}

Como es bien sabido, las lenguas naturales permiten, a diferencia de lo que ocurre con otros sistemas comunicativos, que los hablantes puedan usar el código para hacer referencia al código y, por tanto, hacer uso de la función metalingüística del lenguaje (Jakobson 1975) que se manifiesta a través de la reflexividad lingüística (Lucy 1993).

En esta investigación, el DR se concibe como una categoría lingüística que constituye un continuum (Girón Alconchel 1988 y 2006; Tannen 1989; Semino, Short y Culpeper 1997; Méndez-García de Paredes 2009; Fludernik 2013). Este continuum en el que un texto presente entra en contacto con otro(s) anterior(es) (Reyes 1994) incluye desde la reproducción de citas "literales" hasta la referencia general a otros actos de comunicación.

A partir de un análisis previo del DR en el español caraqueño ( $c f$. Gallucci 2018) hemos identificado, en el marco de ese continuum, seis tipos principales de citas - propias o propiamente de $D R-\mathrm{y}$ dos periféricas -o impropias-. Las primeras incluyen el discurso directo e indirecto con todas sus variantes, la cita mixta, la cita de paralenguaje, la cita de gestos y los ecos. Las segundas, el discurso narrativizado y la cita abstracta ${ }^{4}$.

\section{EL DIÁLOGO RECONSTRUIDO: DEFINICIÓN Y PROPIEDADES}

Como explica Camargo (2007-2008: 50), el diálogo reconstruido

se caracteriza por que cada uno de los turnos de palabra es invariablemente representado en forma de citas por el hablante que, siguiendo la terminología de Goffman (1974 y 1981), anima las distintas voces representadas. Estas animaciones de incrustación múltiple constituyen espectáculos mínimos sobre acciones cotidianas que pueden incluir, además de la representación del discurso de los diferentes personajes

\footnotetext{
4 Hemos denominado a este segundo tipo de citas impropias porque, aunque también en ellas se manifiesta la reflexividad del lenguaje, ello sucede en menor medida que en las anteriores, es decir, aunque estas refieren -más que reproducen- otro discurso, no especifican lo dicho, por lo que su inclusión dentro del DR tradicional (o canónico) es discutible.
} 
cuyo diálogo se cita, las peculiaridades propias de su forma de hablar, de moverse y de gesticular [...] De este modo, los diálogos reconstruidos son también muestras de comunicación multimodal por el uso y la interrelación de diversos modos semióticos de comunicar, esencialmente, el lingüístico, el quinésico y el paralingüístico y, de forma secundaria, el proxémico y el cronémico.

Se trata, entonces, de una instancia polifónica y multimodal (Niemelä 2010, Buchstaller 2014 y 2017) que se manifiesta en la narración oral y en la que el hablante (en nuestro caso, el entrevistado) va ejerciendo alternativamente el rol de emisor y destinatario de turnos de palabra conformados por citas. ${ }^{5}$ Siguiendo a Camargo (2007-2008: 53), hemos caracterizado el DREC según los rasgos básicos relacionados con el análisis conversacional.

En primer lugar, para formar parte de un DREC los enunciados deben ser citas de otros enunciados pasados (posibles o imaginarios), formar una unidad dialogal y constituir secuencias organizadas en pares de pregunta/ respuesta o de aserción/réplica, a lo que puede seguir o no una evaluación.

En segundo lugar, las intervenciones deben responder a la condición de relevancia definida por Schegloff (1972: 76):

When one utterance (A) is conditionally relevant for another (S), then the occurrence of $\mathrm{S}$ provides for the relevance of the occurrence A. If A occurs, it occurs [...] as 'responsive to' S, i.e. in a serial sequenced relation to it.

En tercer lugar, los distintos turnos que componen el diálogo deben representar voces diferentes, es decir, no se consideran DREC los casos de self-talk o de "monólogo interior" en los que el enunciador y el destinatario son correferenciales.

En $(1)^{6}$ ofrecemos un ejemplo de DREC que cumple con los rasgos descritos.

(1) yo le digo <cita $>$ mira / pero ¿cómo es esto que no te mandaron tarea y tienes? </cita $>$ me dice / <cita $>$ bueno / papi / lo que pasa fue

\footnotetext{
5 Camargo (2007-2008: 53) apunta que los DREC son equivalentes al procedimiento retórico de la subiectio y que, incluso, algunos autores han sugerido que estos diálogos constituyen un universal del lenguaje). Más información en este sentido en Carmargo (2010) y Günthner (2002).

6 A fin de facilitar la lectura de los ejemplos, hemos eliminado gran parte del etiquetado de las transcripciones. Hemos incluido únicamente las pausas y las etiquetas que marcan el inicio y el final de una cita.
} 
que cuando tocaron el timbre ya $</$ cita $>/$ yo / <cita $>$ ¡aha! / ¿pero por qué no la hiciste? </cita $><$ cita $>$ bueno / papi / porque me di / no / lo que pasa es que esos niños hacen mucha bulla / papi / y / entonces / uno no se $\operatorname{con}</$ cita $>/<$ cita $>$ bueno / vamos a hacer una cosa / te sientas en la fila de adelante y se arregló el problema $</$ cita $>\left(\right.$ CARA_H11_005) ${ }^{7}$.

\section{DESPLAZAMIENTO Y DIÁLOGOS RECONSTRUIDOS}

Labov y Waletzky (1967) proponen una categoría muy útil para identificar, en las narraciones orales, en qué medida las cláusulas que conforman un relato son dependientes o independientes de la secuencia discursiva en la que aparecen: el desplazamiento. Como la secuencia de tipo narrativo está muy presente en nuestras muestras, precisamente porque los entrevistados han contado, sobre todo, anécdotas e historias que muchas veces se materializan a través de un relato dramatizado, la clasificación de estos autores ha resultado productiva para los fines del presente estudio.

A propósito de esta categoría denominada desplazamiento, los autores distinguen entre cláusulas narrativas o libres. Las cláusulas narrativas dependen de la secuencia discursiva en la que aparecen y, por tanto, no pueden ser desplazadas, es decir, no se podrían mover hacia otras partes de la narración en cuestión, sin ocasionar cambios en la secuencia temporal y lógica de los hechos. Las cláusulas libres, por su parte, son independientes y, por tanto, no están ancladas temporalmente al orden en que se presentan los acontecimientos, esto es, su eventual supresión no generaría cambios sustanciales en el curso del relato.

Aunque hemos utilizado esta clasificación, hemos modificado la nomenclatura: en lugar de cláusulas narrativas y libres, términos que hacen alusión a oraciones y, en consecuencia, a la presencia indispensable de un verbo, nos referiremos a enunciados citativos narrativos o libres (los

\footnotetext{
De aquí en adelante el código especificado al final de los ejemplos debe leerse, de izquierda a derecha, de la siguiente forma: i) $\mathrm{CARA}_{-}=$Caracas; ii) sexo o género: $\mathrm{H}=$ hombres, $\mathrm{M}=$ mujeres; iii) edad: $1=20$ a 34 años, $3=55$ años en adelante; iv) grado de instrucción: 1 = analfabetos o sin estudios, 3 = enseñanza superior; v) identificación del hablante dentro del corpus: 005, 006, 036, 101, etc. Por ejemplo, a través del código CARA_H11_005 se identifica a un hombre caraqueño, de 20 a 34 años, con instrucción primaria, al que le hemos asignado el número 005 en nuestro corpus.
} 
enunciados pueden estar conformados por oraciones o no), pues en el DR el contenido citado no debe ser necesariamente una cláusula.

Los enunciados que hemos denominado narrativos, en el sentido apuntado por Labov y Waletzky (1967), suelen formar parte de un DREC (2). En cambio, los enunciados libres (3) suelen ser casos de DR en los que se reproduce una anécdota ocasional que no está vinculada directamente con los tópicos principales desarrollados en las narraciones ${ }^{8}$ :

(2) Yo vivía con ellos ahí / entonces había un terreno al lado así/ así y él me dijo / <cita $>$ mira ese terreno se está vendiendo / el compadre $\mathrm{F}</$ cita $>$ / porque era un compadre de él / $<$ cita $>$ lo está vendiendo $</$ cita $>$ / tres mil bolívares / <cita $>$ que/ de dónde voy a sacar yo tres mil bolívares $</$ cita $>$ le dije yo en ese tiempo / <cita $>$ no / no / júntate mil bolívares pues / ajúntate mil bolívares y le / y lo demás se lo vas pagando todas las semanas $</$ cita $>$ / todas las semanas le daba unos cinco bolívares / y así me compré el terreno (CARA_H31_029).

(3) pues / me encontré con un lugar donde te enseñan a un Dios mucho más cercano / no es el que conocimos desde que nacemos / que Él está en la iglesia / que ahí no puedes ir en esta forma vestida / que no puedes hacerte tal cosa / que te castiga / que todo es malo / <cita $>$ que Dios te va a juzgar $</$ cita $>$ sino te presentan a un Dios totalmente diferente / un amigo / una persona con quien te puedes entablar conversaciones y sientes respuesta (CARA_M13_084).

Aunque en (2) hemos destacado en negritas la primera cita, es decir, la que sirve para iniciar el diálogo reconstruido que recrea el hablante, las otras, que conforman turnos de habla, también son ejemplos de EDR narrativos.

Por su parte, en (3), si bien hemos destacado el caso de DIL (discurso indirecto libre, es decir, con marco introductor elidido) etiquetado como $<$ cita $>$, los cuatro enunciados anteriores también son casos de cita indirecta sin marco e, igualmente, constituyen ejemplos de enunciados libres. Si prescindimos de estos cinco enunciados citativos, la secuencia que narra el hablante sigue teniendo sentido. En cambio, en (2) todos los EDR que forman parte de ese diálogo que reconstruye el hablante son necesarios. Si eliminamos alguno, los otros perderán sentido.

8 En cada caso ofrecemos el contexto lingüístico necesario. Sin esta información, es prácticamente imposible determinar a qué grupo pertenece cada EDR. 


\section{METODOLOGÍA}

Para llevar a cabo esta investigación, hemos seleccionado una muestra de 16 entrevistas del PRESEEA-Caracas -o Corpus sociolingüistico de Caracas 2004-2013- (Bentivoglio y Malaver 2006, 2012; Gallucci, González y Malaver 2013) estratificadas en cuotas de afijación uniforme según las variables sociales inherentes al corpus.

La distribución por cuotas de los hablantes puede observarse en la tabla 1:

\begin{tabular}{|l|c|c|c|c|c|}
\hline \multicolumn{1}{|c|}{$\begin{array}{c}\text { Grupo } \\
\text { generacional }\end{array}$} & \multicolumn{2}{|c|}{$20-34$ años } & \multicolumn{2}{c|}{55 años y +} & Total \\
\hline Instrucción/Sexo & Hombres & Mujeres & Hombres & Mujeres & \\
\hline Grado 1 & 2 & 2 & 2 & 2 & 8 \\
\hline Grado 3 & 2 & 2 & 2 & 2 & 8 \\
\hline Total & 4 & 4 & 4 & 4 & 16 \\
\hline & \multicolumn{2}{|c|}{8} & \multicolumn{2}{|c|}{8} & \\
\hline
\end{tabular}

Tabla 1. Muestra de análisis

Por tratarse de un estudio piloto sobre los DREC, no hemos seleccionado hablantes con edades comprendidas entre los 35 y los 54 años y, tampoco, aquellos de grado de instrucción 2 (enseñanza secundaria). Nos hemos enfocado en los "extremos" del corpus.

Las transcripciones se han analizado completas en virtud de la riqueza que ofrecen en cuanto al uso del discurso referido. Esto último obedece a la particularidad de las entrevistas: se han tomado como punto de referencia los módulos temáticos propuestos por el PRESEEA, pero el hablante podía desarrollar libremente aquellos temas que quisiera contar o con los que se sintiera más cómodo. Por esta razón, no es de extrañar el elevado número de casos de DR que hemos encontrado en la muestra ( $c f$. Gallucci 2018).

En el análisis nos enfocaremos, pues, en las características (estructura y organización) de los DREC presentes en la muestra (Tannen 1986, 1989). En este caso, hemos considerado cinco aspectos: i) tipos de citas que los conforman, ii) marco introductor de los DREC, iii) turnos, iv) enunciadores o speakers y v) caracterización en función de los factores sociales antes señalados. 


\section{ANÁLISIS}

En primer lugar, hemos extraído de la muestra todos los enunciados de discurso referido y los hemos clasificado según la tipología propuesta por Gallucci (2018). Seguidamente, determinamos, a partir de Labov y Waletzky (1967), su independencia o no en relación con la secuencia discursiva de la que forman parte, es decir, si son libres o narrativos.

Aquellos EDR que resultaron ser "dependientes" o narrativos son los que, efectivamente, forman parte de un DREC. A partir de estos últimos hemos desarrollado el análisis de la estructura y organización de los DREC presentes en las entrevistas.

\subsection{DesPlazamiento y EDR}

Para empezar, nos detendremos en la clasificación de los enunciados de discurso referido según la distinción de Labov y Waletzky (1967) entre enunciados narrativos o dependientes, y enunciados libres o independientes, que ya hemos detallado en $\S 4$.

En la tabla 2 presentamos la distribución de los 2.094 enunciados citativos de nuestra muestra desglosados en función del desplazamiento y el tipo de cita $(\mathrm{CA}=$ cita abstracta, $\mathrm{CG}=$ cita de gestos, $\mathrm{CM}=$ cita mixta, $\mathrm{CP}=$ cita de paralenguaje, $\mathrm{DD}=$ discurso directo, $\mathrm{DI}=$ discurso indirecto, $\mathrm{DN}=$ discurso narrativizado, $\mathrm{E}=\mathrm{ecos})$.

\begin{tabular}{|c|c|c|c|c|c|c|c|c|c|c|}
\hline \multicolumn{2}{|c|}{ Desplazamiento } & \multicolumn{8}{|c|}{ Discurso referido } & \multirow{3}{*}{$\begin{array}{l}\text { Total } \\
1.270\end{array}$} \\
\hline & & CA & CG & $\mathrm{CM}$ & CP & DD & DI & $\mathrm{DN}$ & E & \\
\hline \multirow{2}{*}{ Narrativa } & $\mathrm{N}$ & 1 & 5 & 10 & 10 & 940 & 120 & 184 & 0 & \\
\hline & $\%$ & 0,1 & 0,4 & 0,8 & 0,8 & 74 & 9,4 & 14,5 & 0 & 100 \\
\hline \multirow{2}{*}{ Libre } & $\mathrm{N}$ & 10 & 23 & 9 & 24 & 265 & 141 & 335 & 17 & 824 \\
\hline & $\%$ & 1,2 & 2,8 & 1,1 & 2,9 & 32,2 & 17,1 & 40,7 & 2,1 & 100 \\
\hline \multirow{2}{*}{ Total } & $\mathrm{N}$ & 11 & 28 & 19 & 34 & 1.205 & 261 & 519 & 17 & 2.094 \\
\hline & $\%$ & 0,5 & 1,3 & 0,9 & 1,6 & 57,5 & 12,5 & 24,8 & 0,8 & 100 \\
\hline
\end{tabular}

Tabla 2. DR y desplazamiento 
La tabla 2 muestra que en las entrevistas objeto de estudio los EDR suelen ser narrativos en un $60,6 \%$ de los casos -1.270 ocurrencias- y libres, en un $39,4 \%-824-$.

En cuanto a las tendencias según el tipo de cita, vemos que la CA suele ser libre y, por tanto, su presencia en nuestras entrevistas suele ser anecdótica, es decir, no es imprescindible para que el relato avance, como en (4). Situación similar se refleja en la CP -aunque en menor medida (24 casos de enunciados libres y 10 de narrativos)- y en los E, que siempre son libres. Sobre este último punto, nótese que si suprimimos los ecos de los turnos en los que aparecen, como en (5), se perdería el punto de apoyo del hablante que le permite establecer el tópico y elaborar la respuesta de su intervención, pero no se generaría ningún cambio sustancial en el curso de los acontecimientos que cuenta el entrevistado.

(4) y entonces / practicamos el vocabulario de las cosas del salón de clase / que si lápiz / borra / sacapuntas (CARA_M13_083).

(5) E2: no sé / ¿cómo celebran las Navidades allá? /

I.: ¿las Navidades? / bueno / son muy bonitas / (CARA_H11_005).

En lo que respecta a la $\mathrm{CM}$, vemos que puede ser narrativa o libre prácticamente en la misma proporción: 10 y 9 casos que representan $0,8 \%$ y $1,1 \%$ del total, respectivamente.

En cuanto al DD, los datos reflejan que se trata, la mayoría de las veces ( $940 / 1.205)$, de un enunciado narrativo en los términos establecidos por Labov y Waletzky (1967). Esto nos permite afirmar que los enunciados narrativos de nuestra muestra, es decir, aquellos que no pueden ser desplazados a lo largo de relato conversacional sin traer consigo cambios en la secuencia temporal de los hechos, son, en un $74 \%$, casos de DD. Como apreciaremos en breve, esto se relaciona también con el hecho de que un número importante de estos casos suele formar parte de diálogos reconstruidos en los que los distintos turnos que anima el locutor, en el sentido de Goffman $(1974,1981)$, están anclados temporalmente al orden en que se presentan las distintas voces. Piénsese, por ejemplo, en un par adyacente constituido por una pregunta y una respuesta, como en (6).

(6) le digo <cita> ¿qué pasa K.? // ¿cuánto me depositaron? </cita > $<$ cita $>$ bueno / tú sabes que $</$ cita $>$ / quinientos mil bolívares / (CARA H33_102).

En el DN, la tendencia es inversa, es decir, este tipo de citas sin contenido citado suele ser, al contrario del DD, libre. De hecho, el DN representa el $40,7 \%$ del total de cláusulas libres -335-. Si del fragmento (7) eliminamos 
el caso de DN destacado en negritas, el DREC que reproduce el hablante como locutor, con sus respectivos enunciadores, no se ve alterado:

(7) o salía un día con ella y nos decíamos las cosas / las vainas y que $<$ cita $>$ ¿qué te dijo? $</$ cita $>/<$ cita $>$ no $/ /$ marico que ta $/$ ta $/$ ta $</$ cita $>$ $/<$ cita $>$ ¿sí? $</$ cita $>$ y tal / entonces / hasta que un día le digo $<$ cita $>$ no / no / no / / marico / icon ninguno de los dos! / ¡ con ninguno de los dos! $</$ cita $>$ (CARA_H13_077).

En el DI también se observa cierta inclinación hacia las cláusulas libres, pero muy inferior en relación con el DN; de 261 casos de DI, 141 corresponden a enunciados libres y 120, a narrativos. Esto último puede verse favorecido por el hecho de que la cita indirecta no suele formar diálogos reconstruidos y muchas veces su presencia es accesoria $-\mathrm{O}$ anecdótica- con respecto al desarrollo de las acciones y, por supuesto, de las voces que se reflejan en ellas, como en (8).

(8) los hippies eran unos señores que usaban pantalones bluyins y rotos / como que / que la moda en aquel tiempo / pero eran gente / que eran de riales / de / como se dice hoy en día / los burgueses (CARA_H31_030).

Si del fragmento (8) eliminamos la referencia a los burgueses, que es un caso de discurso cuasi indirecto, no se produce ningún cambio sustancial en la historia que está contando el entrevistado.

\subsection{EDR Y DIÁLOGOS RECONSTRUIDOS}

Ahora bien, en el grupo de los enunciados narrativos que, como hemos visto, suman 1.270 casos que constituyen el $60,6 \%$ del total, debemos destacar los que nos interesan en este trabajo: los EDR que forman parte de un diálogo reconstruido. En nuestra muestra, más de la mitad de los enunciados que han resultado ser narrativos, es decir, 653 están incluidos en un DREC.

Si tomamos como punto de partida el total de casos de nuestra muestra, o sea, 2.094, esas 653 ocurrencias representan el 31,2\%. El resto, es decir, aquellas citas que no se insertan en un DREC, suman 1.441 casos $(68,8 \%)$.

Lo primero que hemos observado en lo que respecta a los DREC ha sido la distribución de los fenómenos del DR que identificamos antes, pero esta vez en función de su inserción o no en un DREC, como se aprecia en la tabla 3. 


\begin{tabular}{|c|c|c|c|c|c|c|c|c|c|c|}
\hline \multirow{2}{*}{\multicolumn{2}{|c|}{ Diálogo reconstruido }} & \multicolumn{8}{|c|}{ Discurso referido } & \multirow{3}{*}{$\begin{array}{c}\text { Total } \\
1.441\end{array}$} \\
\hline & & \multirow{2}{*}{\begin{tabular}{|c|} 
CA \\
11
\end{tabular}} & \multirow{2}{*}{$\begin{array}{c}\mathrm{CG} \\
26\end{array}$} & \multirow{2}{*}{\begin{tabular}{|c|}
$\mathrm{CM}$ \\
15
\end{tabular}} & \multirow{2}{*}{$\begin{array}{l}\mathrm{CP} \\
30\end{array}$} & \multirow{2}{*}{$\begin{array}{l}\text { DD } \\
582\end{array}$} & \multirow{2}{*}{$\begin{array}{c}\text { DI } \\
247\end{array}$} & \multirow{2}{*}{$\begin{array}{l}\mathrm{DN} \\
513\end{array}$} & \multirow{2}{*}{$\begin{array}{c}E \\
17\end{array}$} & \\
\hline & $\mathrm{N}$ & & & & & & & & & \\
\hline & $\%$ & 0,8 & 1,8 & 1 & 2,1 & 40,4 & 17,1 & 35,6 & 1,2 & 100 \\
\hline \multirow{2}{*}{ Insertado } & $\mathrm{N}$ & 0 & 2 & 4 & 4 & 623 & 14 & 6 & 0 & 653 \\
\hline & $\%$ & 0 & 0,3 & 0,6 & 0,6 & 95,4 & 2,1 & 0,9 & 0 & 100 \\
\hline \multirow{2}{*}{ Total } & $\mathrm{N}$ & 11 & 28 & 19 & 34 & 1.205 & 261 & 519 & 17 & 2.094 \\
\hline & $\%$ & 0,5 & 1,3 & 0,9 & 1,6 & 57,5 & 12,5 & 24,8 & 0,8 & 100 \\
\hline
\end{tabular}

Tabla 3. DR y DREC

La tabla 3 muestra que cuando una cita se inserta en un DREC esta es casi siempre directa $(623$ / 653). Esos 623 casos se traducen, en frecuencias relativas, en $95,4 \%$. Esto deja ver que los DREC se construyen básicamente a partir de EDR en DD.

La tabla 3 también evidencia que hay ciertos fenómenos del DR que, en ningún caso, al menos en nuestra muestra, se insertan en un DREC: la CA y el E. El resto de los mecanismos citativos, menos el DI, del que registramos 14 ocurrencias, tiene apenas 6 casos o menos (DN, CP, CM y CG).

En virtud de que los DREC suelen estar formados, como hemos visto, por casos de DD, nos corresponde ver ahora con qué tipo específico de cita directa. Ofrecemos esta información en el gráfico 1.

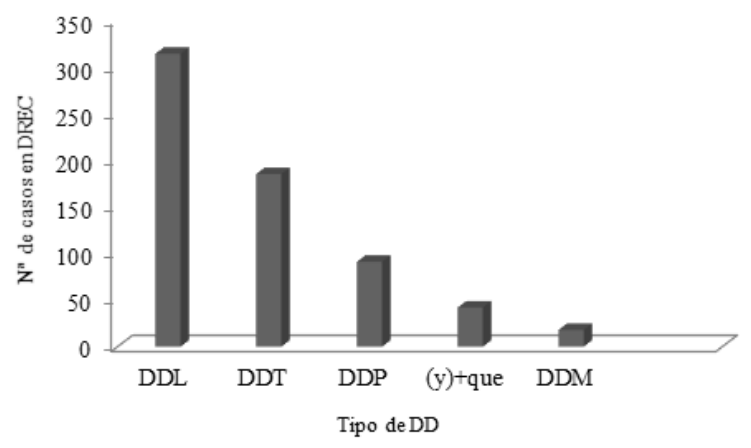

Gráfico 1. DD y DREC

Del gráfico 1 se desprende que el DD suele manifestarse en los DREC especialmente a través de tres formas: i) discurso directo libre o sin marco (DDL) (con 314 casos, 48\%); ii) discurso directo tradicional o con verbo 
de comunicación (DDT) (con 185 ocurrencias, 28\%) y iii) discurso directo con pronombre (DDP) (con 91 enunciados citativos, 14\%). El resto de las formas $-(y)+q u e$ y discurso directo con marcador (DDM)- conforman el $10 \%$ de los casos restantes.

\subsection{MARCO INTRODUCTOR}

Seguidamente, nos ha interesado conocer de qué manera se introducen los DREC en nuestras entrevistas, esto es, cuál es el tipo de cita que se emplea en el primer turno.

El número total de DREC en la muestra del español de Caracas ha sido de 178 diálogos reconstruidos. En el gráfico 2 ofrecemos la distribución de esos 178 DREC en función del marco introductor. En el gráfico solamente aparecen reflejadas ocurrencias de DD, porque los DREC de nuestra muestra solamente se introducen a través del discurso directo.

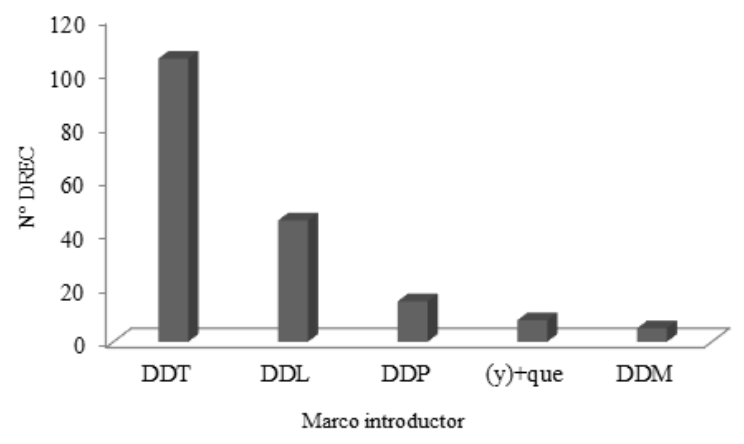

Gráfico 2. Marco introductor de DREC

El gráfico 2 deja ver que el DDT es el marco introductor preferido en los DREC en más de la mitad de los casos (105 ocurrencias que se traducen en $60 \%$ del total). Al DDT le sigue en orden decreciente el DDL, el DDP, $(y)+q u e$ y el DDM con $45(25,3 \%), 15(8,4 \%), 8(4,5 \%)$ y 5 casos $(2,8 \%)$, respectivamente.

A diferencia del gráfico 1, en el que veíamos que cuando se trata de citas que forman parte de un DREC la tendencia más alta se manifiesta en el DDL, que casi duplica al DDT, aquí se invierte la relación y aumenta la frecuencia: cuando se trata de introducir un DREC, el DDT prácticamente triplica al DDL. Esto es esperable en términos de economía del lenguaje. El DDT sirve para marcar claramente que entra en escena una cita, por lo 
que en los EDR siguientes no es obligatorio repetir el marco introductor, a menos que el locutor persiga, a través de la repetición, crear un efecto retórico particular.

Lo último que acabamos de indicar puede apreciarse de manera más clara en el gráfico 3, en el que recogemos, por un lado, las citas en el interior de un DREC -475 casos- $y$, por otro, cuando funcionan como marco introductor (o primer turno) de toda la estructura $-178-$.

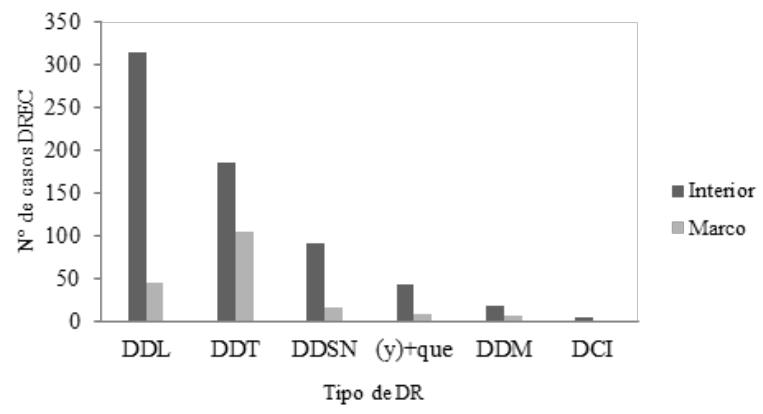

Gráfico 3. Citas en el interior y como marco del DREC

Como explicábamos antes, el gráfico 3 refleja la variabilidad -o inversióna la que hemos hecho referencia, es decir, aquella que se manifiesta especialmente en cuanto al DDL y al DDT. El resto de los fenómenos (DDP, $(y)+q u e$ y DDM) están presentes, en mayor o medida, sobre todo en el interior de un DREC.

\subsection{TURNOS DE HABLA}

Seguidamente, hemos querido conocer el número de turnos que conforman los 178 DREC de nuestras entrevistas. Para que se trate de un DREC, obviamente, deben tener lugar, por lo menos, dos turnos. En estos últimos casos, se trata, por lo general, de pares de pregunta/respuesta o de aserción/ réplica, como en (9) y (10), respectivamente.

(9) I: sí / porque yo estaba en Roma / ya este las demás se habían ido / por razones de trabajo / pero estaba una que se llama Chepina / que es italiana / yo le dije <cita $>$;ay! ¿será que mañana podemos ir? $</$ cita $>/<$ cita $>$ vamos a hacer todo lo posible $</$ cita $>$ / porque ella vive en Sicilia / (CARA_M33_107). 
(10) entonces eso te lo ponían como si fueran galletas y eran algas de láminas / y yo no tenía por qué comérmelas / porque si / si tú querías / te las comías y si no / no / tú te servías / yo comía algas / pusieron unas cosas que eran así como aplastadas // que eran una carne que puede haber sido cerebro de sesos de mono porque tenía como forma y ninguna de la gente del Concejo Central comió eso y / es más / la eslovaca que es médico me dijo < cita $>$ no vayas a comer eso $</$ cita $><$ cita $>$ No $/$ si ya me comí como cuatro / Me encantaron </cita $>$ yo / mira / el picante era súper picante y como a mí me gustan las salsas agridulces y eso / (CARA_M33_107).

Si bien en su mayoría los DREC de nuestra muestra están estructurados de esta manera, o sea, como un par adyacente, hemos encontrado casos con más de dos turnos. Esta información la hemos reflejado en el gráfico 4.

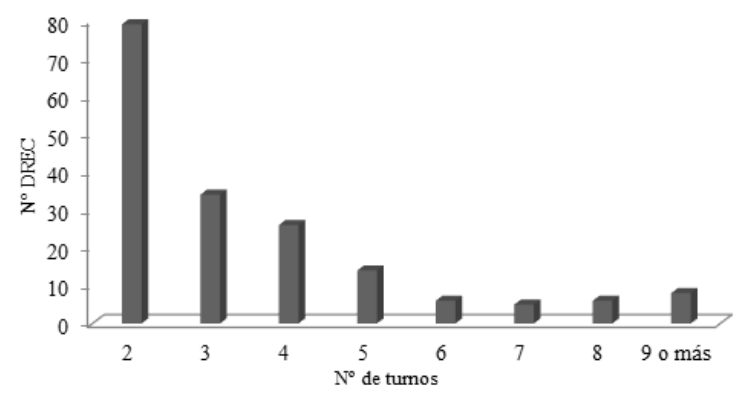

Gráfico 4. DREC según número de turnos

En el gráfico 4 es posible apreciar que 79 DREC son de 2 turnos, 34 de 3, 26 de 4 y 14 están constituidos por 5 turnos. El resto de las opciones $(6,7$ y 8 turnos) agrupa menos de 6 DREC en cada caso. Los diálogos reconstruidos que agrupamos bajo la etiqueta ' 9 o más', como su nombre indica, recogen aquellos DREC que tienen más de 9 de turnos. En este grupo solamente encontramos 2 DREC con 9 turnos. El resto está conformado por DREC de $11,12,13,14,15$ y 16 turnos, de los que hemos registrado una ocurrencia de cada caso; de allí que el total de DREC en este grupo tenga una frecuencia absoluta de 8 .

A continuación, reproducimos el DREC con mayor número de turnos de nuestra muestra -16-. Nótese que el DREC que presentamos en (11) no se ve interrumpido por las intervenciones del entrevistador (E1), quien muestra que está siguiendo con interés el diálogo que reproduce el entrevistado, tal como se desprende de los distintos comentarios de tipo fático que hace. El 
entrevistado asume el papel de locutor y, cuando se cita a sí mismo, de otro enunciador.

(11) él fumaba muchísimo / eso era una cosa que ¡compulsiva! ¡ese humero! / una cosa horrible y hablaba así todo rapidito / argentino / entonces me dijo <cita> siéntese ahí / ¿qué piensa estudiar usted? </ cita $><$ cita $>$ Bueno / mire / yo pensaba estudiar economía / me gusta la matemática $</$ cita $>$ me dice $<$ cita $>$ mire / usted no puede estudiar nada de eso $</$ cita $><$ cita $>$ ¿y por qué no? $</$ cita $><$ cita $>$ abra ese sobre $</$ cita $>$ yo abro el sobre / me dice $<$ cita $>$ el resultado suyo en la parte numérica son completamente anormales $</$ cita $>$ ¡Te puedes imaginar esa expresión para un muchacho de diecisiete años! / bueno $<$ cita $>$ si son completamente anormales / usted es tan anormal que raya en la imbecibilidad $</$ cita $>$ / E1: ¿qué?

I: entonces ya coño / me estaba subiendo / < cita > no se moleste / no se moleste / usted / hablando en argentino criollo / en venezolano / usted es bruto / usted es prácticamente una bestia $</$ cita $>$ /

E1: ¡no puede ser! /

I: sí / así / clarito / yo en eso me paré < cita $>$ si usted estudia algo de eso / usted nunca se va a graduar y se va a acordar de mí / abra este sobre $<$ / cita $>$ la parte verbal / todos los números ahí bastante grandes y entonces me dice <cita $>$ en la parte verbal usted también es anormal / usted es completamente anormal $</$ cita $>$ yo estuve a punto de pararme y salirme $<$ cita $>$ pero / espérese un momentito / es que usted está por encima de lo normal $</$ cita $>$ / la cosa me /

E1: $<$ ininteligible/ $>$ /

I: $<$ cita $>$ usted tiene que estudiar carreras humanísticas / preferiblemente historia </cita $>$ / < cita $>$ ¡no profesor! ¡usted está loco! / mire / yo en historia siempre me porto mal para que me boten de clase / me pongo a jugar básquet / a jugar voleibol / ponerme en cuestiones políticas / hacer pancartas / bueno / leer ni hablar / eso es fastidiosísimo / además esas son cosas que ya pasaron / a mí no me gusta eso / usted está loco / usted está loco / usted está tostado completamente $</$ cita $>$ / $<$ cita $>$ no / no / mire / usted tiene que estudiar historia / puede estudiar también literatura / usted es humanista $</$ cita $>/<$ cita $>$ no / ¡yo soy de ciencias! / yo nunca quise estudiar humanidades $</$ cita $>$ / $<$ cita $>$ si usted estudia carreras humanísticas usted va a ser un éxito total / usted verá que todo será fácil / tenga su sobre / firme aquí / hasta luego $</$ cita $>$ entonces me fui así tú sabes dándole vueltas al asunto (CARA_H33_101). 


\subsection{ENUNCIADORES}

Además del número de turnos, hemos querido conocer el número de enunciadores o speakers en cada uno de los 178 DREC de la muestra. Tannen (1986: 319) ha afirmado que en la oralidad los diálogos reconstruidos no están limitados a dos voces, como suele ocurrir tradicionalmente en la escritura. En el gráfico 5 ofrecemos los resultados que obtuvimos en ese sentido.

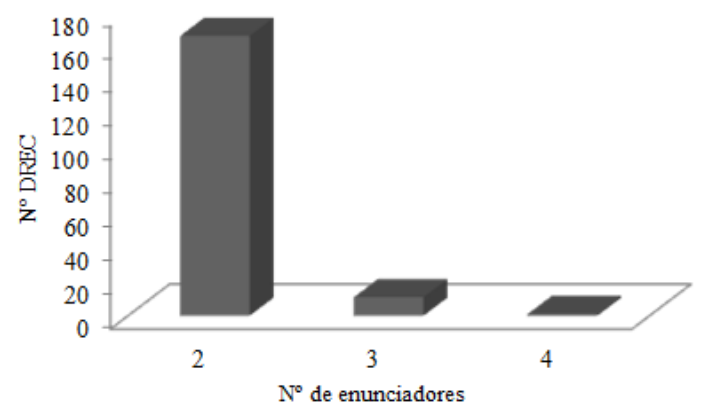

Gráfico 5. Enunciadores y DREC

El gráfico 5 deja ver, sin lugar a duda, que en los DREC de nuestras entrevistas el locutor suele animar, en su mayoría, dos voces -166 casos-, incluida la propia, como en (12); aunque se trate de la oralidad y el hablante tenga la posibilidad de reproducir un mayor número de voces, en consonancia con lo apuntado por Tannen (1986) ${ }^{9}$. Obviamente, esto dependerá también del número de participantes involucrados en las historias que relatan los entrevistados.

(12) y ella me decía / <cita > empuja </cita $>$ y yo / <cita > jno! / jno! $</$ cita $>$ / pues / yo tenía mucho miedo / (CARA_M11_011).

Debemos destacar, igualmente, desde el punto de vista conversacional, que prácticamente todos los EDR registrados, incluidos los que sirven de marco del DREC, son introducidos por el propio interlocutor responsable de la narración y son, por tanto, monológicos. Como apunta Briz (2016: 53), "el relato se acomoda dentro de la estructura de la conversación. Su carácter

\footnotetext{
9 Sin embargo, en este punto debemos acotar que si incluimos al locutor (o narrador) serían, en realidad, tres voces. En el ejemplo (12) tendríamos entonces: i) la voz de la entrevistada como locutor que narra los hechos, ii) la voz de la participante femenina identificada a través del pronombre ella (enunciador i); y iii) la voz de la entrevistada en la situación anterior que se está recreando (enunciador ii).
} 
prototípicamente monológico no contradice su carácter interaccional (no en vano, las historias reproducen casi siempre diálogos)"10.

La presencia de tres enunciadores en un DREC se registra en apenas 11 ocasiones.

\subsection{VARIABLES SOCIALES}

A la par de todo lo que hemos comentado hasta ahora a propósito de los DREC, y retomando el hecho de que los distintos fenómenos del DR que hemos identificado en nuestra muestra pueden estar insertados o no en un diálogo de este tipo, a continuación, ofrecemos estos resultados en relación con las variables extralingüísticas inherentes a la muestra (edad, sexo y grado de instrucción). Las tablas que siguen incluyen los resultados del $\chi^{2}$ y el $p$ valor que hemos obtenido a través del programa SPSS Statistics 22 .

\subsubsection{Edad}

En la tabla 4 pueden observarse las frecuencias absolutas y relativas de los fenómenos del DR que se insertan o no en un DREC, a manera de variable dependiente, en relación con el factor 'edad' como variable independiente.

\begin{tabular}{|c|c|c|c|c|}
\hline \multirow{2}{*}{\multicolumn{2}{|c|}{ Edad }} & \multicolumn{2}{|c|}{ Diálogo reconstruido } & \multirow{3}{*}{$\begin{array}{r}\text { Total } \\
1.174 \\
\end{array}$} \\
\hline & & \multirow{2}{*}{$\begin{array}{c}\text { Insertado } \\
381\end{array}$} & \multirow{2}{*}{$\begin{array}{c}\text { No } \\
\text { insertado } \\
793\end{array}$} & \\
\hline 20 a 34 & $\mathrm{~N}$ & & & \\
\hline años & $\%$ & 32,5 & 67,5 & 100 \\
\hline \multirow{2}{*}{$\begin{array}{c}55 \text { años } \\
\text { y más }\end{array}$} & $\mathrm{N}$ & 272 & 648 & 920 \\
\hline & $\%$ & 29,6 & 70,4 & 100 \\
\hline \multirow{2}{*}{ Total } & $\mathrm{N}$ & 653 & 1.441 & 2.094 \\
\hline & $\%$ & 31,2 & 68,8 & 100 \\
\hline \multicolumn{2}{|c|}{$\chi^{2}=2,0051$ g.d. } & $(3,841)$ & \multicolumn{2}{|c|}{$p=0,157$} \\
\hline
\end{tabular}

Tabla 4. DREC y edad

10 En este punto debemos aclarar que lo hemos planteado de esta manera, pues las entrevistas del PRESEEA-Caracas están a medio camino entre la entrevista semidirigida y la conversación, en virtud de lo que como comentamos en $\$ 5$ a propósito de la metodología que seguimos en la recolección de las muestras (protagonismo del entrevistado y de sus temas de interés). 
Los resultados muestran que no hay mayores diferencias en cuanto a los hablantes jóvenes $(32,5 \%)$ o mayores $(29,6 \%)$ en el uso de citas que forman parte de un DREC. Según la prueba del $\chi^{2}$, no hay probabilidad $(p=0,157>$ 0,050 ) de que la edad de los hablantes incida en la inserción o no de un EDR en un DREC, ya que el valor muestral $(2,005)$ no supera al esperado $(3,841)$.

Camargo (2007-2008: 64), en cambio, obtuvo significatividad estadística en el caso de esta variable. Ambos estudios coinciden, no obstante, en que los DREC se usan más en el grupo etario entre 20 y 34 años (21 a 35 años en la muestra de esta última autora) (Gallucci 2018: 374).

\subsubsection{Sexo}

El análisis de los fenómenos del DR que se insertan o no en un DREC en relación con la variable 'sexo' proporciona los resultados que aparecen en la tabla 5:

\begin{tabular}{|c|c|c|c|c|c|}
\hline \multicolumn{3}{|c|}{ Sexo } & \multicolumn{2}{|c|}{ Diálogo reconstruido } & \multirow{3}{*}{$\begin{array}{l}\text { Total } \\
1.089\end{array}$} \\
\hline & & & Insertado & No insertado & \\
\hline & \multirow[t]{2}{*}{ Mujeres } & $\mathrm{N}$ & 245 & 844 & \\
\hline & & $\%$ & 22,5 & 77,5 & 100 \\
\hline & \multirow[t]{2}{*}{ Hombres } & $\mathrm{N}$ & 408 & 597 & 1.005 \\
\hline & & $\%$ & 40,6 & 59,4 & 100 \\
\hline \multirow{2}{*}{\multicolumn{2}{|c|}{$\begin{array}{c}\text { Total } \\
\%\end{array}$}} & $\mathrm{~N}$ & 653 & 1.441 & 2.094 \\
\hline & & 31,2 & 68,8 & 100 & \\
\hline \multicolumn{2}{|c|}{$\chi^{2}=79,784$} & $1 g$. & 1. $(3,841)$ & $p=0,000$ & \\
\hline
\end{tabular}

Tabla 5. DREC y sexo

A diferencia de la edad, en esta oportunidad los valores relativos observados indican que son los hombres $(40,6 \%)$, y no las mujeres $(22,5 \%)$, quienes suelen emplear más EDR en el marco de un DREC y, en consecuencia, los que contribuyen al uso de este último en la muestra.

El resultado de la prueba del $\chi^{2}$ permite rechazar la hipótesis nula -es decir, aquella que establece que dos variables no están relacionadas-, pues su valor muestral $(79,784)$ es superior al valor esperado; de la misma manera, el $p$ valor $(0,000)$ es menor al límite establecido para los estudios lingüísticos $(p<0,050)$. En cuanto al tamaño del efecto (TE) o Phi $(\phi)$, en este caso obtuvimos un valor de .195 que, en los valores referenciales a propósito de la prueba del chi cuadrado $(.10=$ pequeño, $.30=$ mediano y $.50=$ grande $)$, representa que la magnitud del efecto es pequeña. 
Los datos obtenidos aquí son opuestos a los de Camargo (2007-2008), quien encuentra que las mujeres usan más los DREC que los hombres $(85 \%$ vs. $15 \%$ ). En este punto es importante recordar que el corpus que emplea Camargo corresponde al Atlas Lingüístico (y etnográfico) de Castilla-La Mancha (ALeCMan), que incluye entrevistas semidirigidas con tres grados de formalidad (máxima, media y mínima), y el Corpus Espontáneo recogido por la autora (Camargo 2004) con grabadora oculta en situaciones familiares e informales. Nuestras entrevistas son semidirigidas / conversacionales, y con grabadora a la vista (Gallucci 2018: 375-376).

\subsubsection{Grado de instrucción}

En la tabla 6 ofrecemos los resultados sobre los DREC y el grado de instrucción de los hablantes.

\begin{tabular}{|c|c|c|c|c|}
\hline \multirow{2}{*}{\multicolumn{2}{|c|}{ Grado de instrucción }} & \multicolumn{2}{|c|}{ Diálogo reconstruido } & \multirow{3}{*}{$\begin{array}{r}\text { Total } \\
894\end{array}$} \\
\hline & & \multirow{2}{*}{$\begin{array}{c}\text { Insertado } \\
183\end{array}$} & \multirow{2}{*}{$\begin{array}{c}\text { No insertado } \\
711\end{array}$} & \\
\hline Dim & $\mathrm{N}$ & & & \\
\hline Primlanta & $\%$ & 20,5 & 79,5 & 100 \\
\hline \multirow{2}{*}{ Universitaria } & $\mathrm{N}$ & 470 & 730 & 1.200 \\
\hline & $\%$ & 39,2 & 60,8 & 100 \\
\hline \multirow{2}{*}{ Total } & $\mathrm{N}$ & 653 & 1.441 & 2.094 \\
\hline & $\%$ & 31,2 & 68,8 & 100 \\
\hline \multicolumn{2}{|l|}{$\chi^{2}=83,456$} & g.d.l. $(3,841$ & $p=0,0$ & \\
\hline
\end{tabular}

Tabla 6. DREC y grado de instrucción

Las frecuencias absolutas y relativas correspondientes a la inserción de un EDR en un DREC y la variable extralingüística 'grado de instrucción' muestran que son los hablantes de grado de instrucción universitaria los que contribuyen en este sentido: 470 casos que equivalen al $39,2 \%$ frente a $183(20,5 \%)$ de los de instrucción primaria. La aplicación de la prueba del $\chi^{2}$ indica que el grado de instrucción del hablante incide en la inserción o no de un fenómeno del DR en un DREC $(p=0,000<0,050)$, pues el valor experimental $(83,456)$ es superior al teórico $(3,841)$. Esto nos permite rechazar la hipótesis nula (H0). Como en el caso anterior, es decir, el de la variable 'sexo', la magnitud del efecto también ha sido pequeña $(\phi=.20)$, aunque ligeramente superior a la primera. 
A propósito del grado de instrucción, Camargo (2007-2008: 65) encuentra que el uso del DR en los DREC disminuye a medida que sube el grado de instrucción. En nuestro caso es al revés, aunque la diferencia entre el grado de instrucción primaria y el universitario ( $20,5 \%$ vs. $39,2 \%)$ no es tan marcada como muestran los datos de la autora (34\%vs. 4\%). Debemos recordar aquí lo que apuntábamos antes sobre las características de las muestras de Camargo y la nuestra. Igualmente, que analizamos 16 entrevistas y la autora, 12; lo que no nos permite establecer una comparación en sentido estricto entre ambos estudios (Gallucci 2018: 377-378) ${ }^{11}$.

\section{CONCLUSIONES}

Los resultados generales obtenidos permiten constatar que, en nuestra muestra, los DREC están conformados, sobre todo, por citas directas sin marco o freestanding quotations (Cameron 1998). Asimismo, que suelen estar introducidos por citas directas tradicionales, es decir, encabezadas por un verbo dicendi conjugado.

En lo que respecta a la organización interna de los DREC, hemos visto que en su mayoría están constituidos por secuencias organizadas en pares de pregunta/respuesta o de aserción/réplica, aunque hemos registrado un diálogo reconstruido de 16 turnos.

Asimismo, a pesar de que Tannen (1986: 319) ha afirmado que en la oralidad, a diferencia de la escritura, los diálogos reconstruidos no están limitados a dos voces, en nuestras entrevistas el locutor suele animar, en su mayoría, dos voces, incluida la propia. Como hemos apuntado antes, esto guarda estrecha relación con el número de participantes presentes en las historias que narran los hablantes. También podría estar vinculado con las particularidades de las entrevistas objeto de estudio (con un número limitado de participantes).

En lo que respecta a las variables sociales, los datos estadísticos de este estudio piloto muestran que el uso de EDR en el marco de un DREC guarda

11 En lo que tiene que ver con los factores sociales, hemos comparado nuestros resultados únicamente con los de Camargo (2007-2008), pues ambos estudios están centrados en los DREC. A propósito del uso del discurso referido en el marco del PRESEEA, y su relación con las variables sociales en distintas variedades del español, véase Gallucci (2018: 378-385). 
relación, al menos en nuestras entrevistas, con el sexo y la instrucción de los hablantes. No obstante, hay que recordar que, en todos los casos, la magnitud del efecto es pequeña, lo que no permite hacer afirmaciones concluyentes en este sentido.

Los resultados comentados en cada categoría lingüístico-pragmática también han dejado ver de qué manera en las narraciones orales el discurso referido y sus variantes se articulan a través de ciertos patrones recurrentes (discurso directo libre en el interior, discurso directo tradicional como marco introductor, dos turnos y dos voces que se animan).

Además de ampliar la muestra e incluir datos de distintas variedades dialectales, en futuras investigaciones sería interesante analizar entrevistas filmadas que permitan estudiar la multimodalidad inherente a los diálogos reconstruidos, aspecto fundamental que, por la configuración intrínseca de las entrevistas del PRESEEA, excede los límites de este trabajo.

\section{REFERENCIAS BIBLIOGRÁFICAS}

Benavent Payá, Elisa. 2015. Decir y discurso directo en los relatos de la conversación coloquial. Tesis doctoral, Universitat de València, Valencia-España.

Bentivoglio, Paola e Irania Malaver. 2006. La lingüística de corpus en Venezuela: un nuevo proyecto. Lingua Americana 19: 37-46.

2012. Corpus Sociolingüístico de Caracas: PRESEEA 2004-2010. Hablantes de instrucción superior. Boletín de Lingüistica XXIV, 37-38: 144-180.

BRIZ, ANTONIO. 2016. El relato coloquial: un hecho conversacional narrativo y una estrategia. En Elena Carpi; Rosa García y Elena Liverani. Le forme del narrare: nel tempo e tra $i$ generi. Vol. I, pp. 7-60. Trento: Università degli Studi di Trento.

Buchstaller, Isabelle. 2014. Quotatives: New Trends and Sociolinguistic Implications. New Jersey: Wiley-Blackwell.

2017. Reported speech. En Anne Barron; Peter Grundy y Yueguo Gu Yueguo (eds.). The Routledge Handbook of Pragmatics, pp. 399-417. Oxford: Routledge.

Camargo, Laura. 2007-2008. La cita como turno: el diálogo reconstruido en español oral desde la pragmática del corpus. Pragmalinguistica 15-16: 49-70.

2010. Dialogues within oral narratives: Functions and forms. En April Dale y Lidia Rodríguez-Alfano (eds.). Dialogue in Spanish. Studies in functions and contexts, pp. 3154. Amsterdam: John Benjamins.

CAMERon, Richard. 1998. A variable syntax of speech, gesture, and sound effect: Direct quotations in Spanish. Language Variation and Change 10: 43-83.

Estévez Rionegro, Noelia. 2016. Las construcciones de estilo directo en español. Estudio de corpus. Tesis doctoral, Universidade de Santiago de Compostela, Santiago de Compostela.

Fernández, María Fernanda. 2012. Discurso directo e indirecto en el español de Mérida. Lengua y Habla 16: 71-85. 
FLuderniK, MoniKa. 2013. Conversational Narration - Oral Narration. En Peter Hühn et al. (eds.). The living handbook of narratology. Hamburg: Hamburg University. Disponible en http://www.lhn.uni- hamburg.de/article/conversational-narration-oral-narration [Consulta: 5 de abril de 2020].

GallucCi, María José. 2013. Más sobre el estilo directo e indirecto en el español de Caracas. Lengua y Habla 17: 89-111.

2014. Entonces un muchacho llega y me dice: "Mira, regáleme un bolivar". Las citas en estilo directo e indirecto en el Corpus diacrónico del habla de Caracas 1987/2013. Boletín de Lingüistica XXVI, 41-42: 43-72.

2018. Contribución al estudio del discurso referido en un corpus oral del español americano. Tesis doctoral, Universidad de Zaragoza.

2019. Los verbos introductores de cita en la narración oral conversacional. En Adrián Cabedo Nebot y Antonio Hidalgos (eds.). Pragmática del español hablado. Hacia nuevos horizontes, pp. 29-42. Valencia: Universitat de València.

Gallucci, María José; Carla González e Irania Malaver. 2013. Corpus sociolingüístico "PRESEEA Caracas 2004-2010". Hablantes de grado 1 de instrucción. Boletín de Lingüística XXV, 39-40: 92-107.

Gallucci, María José y Kerlys Vargas. 2015. Estilo directo e indirecto en el Corpus sociolingüístico de Caracas 1987. Anuario de Letras III (2): 65-103.

Girón AlCONChel, José Luis. 1988. La reproducción del discurso en la lengua hablada. II Simposio Internacional de Semiótica. Lo cotidiano y lo teatral, vol. I., pp. 203-215. Oviedo: Servicio de Publicaciones de la Universidad de Oviedo.

2006. Sobre la gramaticalidad del discurso referido. En Concepción Company (ed.). El español en América. Diatopía, diacronía e historiografía. Homenaje a José Moreno de Alba, pp. 395-406. México, DF: Universidad Nacional Autónoma de México.

Goffman, ERving. 1974. Frame analysis: An essay on the organization of experience. Boston: Northeastern.

1981. Forms of Talk. Oxford: Blackwell.

Grajales, Róbinson. 2017. El discurso referido en el español de Medellín, Colombia. Lenguaje 45 (2): 221-246.

GüNTHner, Susanne. 2002. Perspectivity in reported dialogues. The contextualization of evaluative stances in reconstructing speech. En Carl Graumann y Werner Kallmeyer (eds.). Perspectivity and Perspectivation in Discourse, pp. 347-374. Amsterdam: John Benjamins.

JAKOBSON, Roman. 1975. Ensayos de lingüística general. Barcelona: Seix Barral.

Klee, Carol y Rocío CARAVEdo. 2017. El discurso referido en la narración oral de hablantes de Lima. Comunicación presentada en el XVIII Congreso Internacional de la ALFAL. Bogotá, 24-28 de julio de 2017.

Labov, William y Joshua Waletzky. 1967. Narrative analysis: Oral versions of personal experience. En June Helm (ed.). Essays on the verbal and visual arts: Proceedings of the 1996 Annual Spring Meeting of the American Ethnological Society, pp. 12-44. Seattle: University of Washington Press.

LuCy, John (ed.). 1993. Reflexive language. Reported speech and metapragmatics. Cambridge: Cambridge University Press.

Méndez-García de Paredes, Elena. 2009. Los enunciados del discurso referido y la gramática. En María Victoria Camacho; José Javier Rodríguez y Juana Santana (eds.). Estudios de lengua española: descripción, variación y uso. Homenaje a Humberto López Morales, pp. 483-517. Iberoamericana /Vervuert: Frankfurt am Main.

Niemelä, MAARIT. 2010. The reporting space in conversational storytelling: Orchestrating all semiotics channels for taking a stance. Journal of Pragmatics 42: 3258-3270. 
Pascual, Esther. 2014. Fictive Interaction: The Conversation Frame in Thought, Language, and Discourse. Amsterdam / Philadelphia: John Benjamins.

Pascual, Esther y Sergeiy Sandler (eds.). 2016. The Conversation Frame: Forms and Functions of Fictive Interaction. Amsterdam / Philadelphia: John Benjamins.

REPEDE, DoINA. 2018. El uso del discurso referido en el corpus PRESEEA-Sevilla: el sociolecto alto. Anuario de Letras VI (1): 187-218.

Reyes, Graciela. 1994. Los procedimientos de cita: citas encubiertas y ecos. Madrid: Arco/ Libros.

San Martin, Abelardo. 2015. Variación sintáctica y discursiva en el español hablado en Santiago de Chile. Análisis sociolingüístico del queísmo, el discurso referido y los marcadores de reformulación. Tesis doctoral, Universidad de Valladolid, Valladolid.

San Martín, Abelardo y Silvana Guerrero. 2013. Una aproximación sociolingüística al empleo del discurso referido en el corpus PRESEEA de Santiago de Chile. Revista Signos 46 (82): 258-282.

SCHEGLOFF, EMANUEL. 1972. Notes on a conversational practice: formulating place, En David Sudnow (ed.), Studies in Social Interaction, pp. 75-119. New York: Free Press.

Semino, Elena; Mick Short y Jonathan Culpeper. 1997. Using a Corpus to Test a Model of Speech and Thought Presentation. Poetics 5: 17-43.

Tannen, Deborah. 1986. Introducing constructed dialogue in Greek and American conversational and literary narrative. En Florian Coulmas (ed.). Direct and indirect speech, pp. 311-332. Berlín: Mouton de Gruyter.

1989. Talking voices: Repetition, dialogue and imagery in conversational discourse. Cambridge: Cambridge University Press. 